OPEN ACCESS

Edited by:

Zhi Sheng,

Virginia Tech, United States

Reviewed by:

Min Hee Kang,

Texas Tech University Health Sciences

Center, United States

Jian-ye Zhang,

Guangzhou Medical University, China

Sujuan Guo,

Dana-Farber Cancer Institute,

United States

*Correspondence:

Zan Shen

sshenzzan@vip.sina.com

Specialty section:

This article was submitted to

Cancer Molecular Targets and

Therapeutics,

a section of the journa

Frontiers in Oncology

Received: 25 July 2018 Accepted: 11 December 2018

Published: 04 January 2019

Citation:

Yu W, Tang L, Lin F, Yao Y and Shen Z (2019) DGKZ Acts as a Potential

Oncogene in Osteosarcoma Proliferation Through Its Possible Interaction With ERK1/2 and MYC

Pathway. Front. Oncol. 8:655. doi: 10.3389/fonc.2018.00655

\section{DGKZ Acts as a Potential Oncogene in Osteosarcoma Proliferation Through Its Possible Interaction With ERK1/2 and MYC Pathway}

\author{
Wenxi Yu, Lina Tang, Feng Lin, Yang Yao and Zan Shen* \\ Department of Oncology, Affiliated Sixth People's Hospital, Shanghai Jiaotong University, Shanghai, China
}

Osteosarcoma (OS) is one of the most common primary bone tumors in children and young adults. The majority of osteosarcoma patients have limited alternative therapeutic options and metastatic patients generally have a poor prognosis. Thus, it is important to explore novel effective therapeutic targets in the treatment of osteosarcoma. Diacylglycerol kinase zeta (DGKZ) is a recently identified gene potentially associated with certain human carcinogenesis. However, the role of DGKZ in proliferation of osteosarcoma is still unclear. In this study, DGKZ's expression was firstly investigated in OS tumor samples and correlated with poor outcome in OS patients. Silence of DGKZ by shRNA hampered osteosarcoma cell growth and promoted cell apoptosis in vitro. In vivo, DGKZ's knockout also suppressed xenograft tumor proliferation as determined by bioluminescence imaging and weight/volume measurements. Meanwhile, Affymetrix GeneChip and Ingenuity Pathway Analysis (IPA) revealed that DGKZ knockdown resulted in a decreased activity of MYC pathway, and several target genes expression in MYC pathway were altered, including CCND1, CDKN2B, CDK6, PCNA, and EGR1. Furthermore, immunoprecipitation coupled with mass spectrometry (IP-MS) analysis was used to identify proteins that interacted with DGKZ in OS cells and revealed ERK1/2, a key MYC-interactor, to associate with DGKZ. Together, our study demonstrated that DGKZ might act as an oncogene in osteosarcoma via its possible interaction with ERK1/2 and MYC pathway.

Keywords: osteosarcoma, DGKZ, oncogene, ERK1/2, MYC

\section{INTRODUCTION}

Osteosarcoma is the most common aggressive form of bone tumor occurs in children and young adults $(1,2)$. Traditional therapeutic approaches include local control of the primary lesion by surgery and/or chemotherapy, and treatment of disseminated disease with multiagent cytotoxic.

Chemotherapy (3), Somehow, patients those with metastatic or recurrent disease have extremely poor survival rates even after normative chemotherapy and curative resection of the primary lesion (4-6). Hence, there is urgent need for development of new therapeutic approaches and a further investigation in proliferation of osteosarcoma. However, progression of osteosarcoma is associated with numerous genes with diverse genomic alterations and the molecular carcinogenesis of osteosarcoma has not been fully elucidated (7). 
Diacylglycerol (DG) is an important second messenger involved in a variety of cellular responses including proliferation, differentiation, motility, and secretion $(8,9)$ which could activate various effector proteins. Intracellular DAG levels have been shown to play a vital role in cellular growth responses (10). Thus, levels of DG should be maintained strictly to keep cellular environment within a physiological range. Diacylglycerol kinases (DGK) are a class of enzymes that convert DAG into PA and act as key modulators of DG levels (11). Recent studies have found 10 isozymes of DGK, each showing unique tissue expression and distinct subcellular localization (12-15).Among the 10 isoforms, diacylglycerol kinase zeta (DGKZ) is the only one that contains a nuclear localization signal $(16,17)$ and has proven to be associated with various physiological or pathophysiological signaling pathways $(16,18-25)$. Recent studies have proven that in colon cancer and gliomas, DGKZ plays an key role in oncogenesis. (26-28). Additionally, DGKZ also showed its function in regulating bone homeostasis through osteoclasts via modulation of c-Fos (20), which is a key regulator in proliferation of osteosarcoma (29-32). However, the potential role of DGKZ in oncogenesis of osteosarcoma is still unclear.

In our study, DGKZ's expression was upregulated in osteosarcoma tissues and correlated with patients' poor prognosis. In vitro, DGKZ knockdown impaired proliferation and promoted apoptosis in osteosarcoma cells. In vivo, DGKZsilencing xenografts in nude mice formed smaller tumors while compared with control group. Additionally, microarray and IPA analyses demonstrated a alternation in expression of signaling-related genes in osteosarcoma cells and verified MYC pathway as a direct downstream target of DGKZ. In addition, association between DGKZ and ERK1/2, a key MYC-interactor, was verified through immunoprecipitation-mass spectrometry (IP-MS) approach. These results suggest a potential interaction between DGKZ and ERK1/2-MYC pathway might play an important role in promotion of osteosarcoma, indicating that interfering with function or expression of this interaction may be a potential route to block the invasiveness of osteosarcoma.

\section{MATERIALS AND METHODS}

\section{Ethics Statement}

The Ethics Committees of Affiliated Sixth People's Hospital, Shanghai Jiaotong University has approved all studies about human participants. Informed and written consents were obtained from all patients or their advisers according to ethics committee guidelines.

\section{Patient Samples and Follow-Up}

Tumor specimens from 80 patients suffered from IIB limb osteosarcoma between 2012 and 2015 and 24 cases of normal bone tissues as control group were collected from Affiliated Sixth People's Hospital, Shanghai Jiaotong University for this study. The normal bone tissues were resected within at least $5 \mathrm{~cm}$ to the margin of tumor while definitive surgery was administrated. After surgical resection, all the osteosarcoma and normal bone tissues were frozen within $30 \mathrm{~min}$. All patients were treated with combination chemotherapy which consist high doses of methotrexate, cisplatin, ifosfamide, and doxorubicin, then underwent local resection of the primary tumor, followed by adequate cycles of adjuvant chemotherapy. After completion of chemotherapy,

patients were followed every 3 months for 4 years and then every 6 months for 10 years.

\section{Immunohistochemical Staining of DGKZ in OS Samples}

Paraffin sections were treated with hydrogen peroxide to inactivate endogenous peroxidases. Antigen retrieval was performed in a microwave in $10 \mathrm{mmol} / \mathrm{L}$ citrate buffer at $\mathrm{pH}$ 6.0. Sections were then incubated in anti-DGKZ antibody overnight at $4{ }^{\circ} \mathrm{C}$, and a secondary antibody was used to detect protein expression (27). For each run of immunohistochemistry, appropriate positive and negative controls were performed. Immunohistochemistry staining was assessed by two blinded independent pathological observers who were blinded to patient identity. Percentage of osteosarcoma cells stained positive for DGKZ was recorded, with 0 denoting none of osteosarcoma cells stained, 1 denoting 1-25\% of osteosarcoma cells stained, 2 denoting $26-50 \%$ of osteosarcoma cells stained, 3 denoting $51-75 \%$ of osteosarcoma cells stained, and 4 denoting $76-100 \%$ of osteosarcoma cells stained. Intensity of nuclear/cytoplasm/cytomembrane staining on a scale was also recorded, with 0 denoting no staining, 1 denoting slight/weak staining, 2 denoting strong staining; and 3 denoting intense staining. Score was calculated as percentage of cells stained positive multiplied by intensity of staining, and was defined as positive (score above 6) or negative( score $\leq 6)$.

\section{Cells Lines and Cell Culture Conditions}

Osteosarcoma cell lines that included SaoS-2, U-2OS, HOS, MG63, human normal osteoblasts hFOB1.19 cell line and human renal epithelial 293T cell line were obtained from China Center for Type Culture Collection (CCTCC, Shanghai, China). Cells were grown in Dulbecco's modified Eagle's medium, Eagle's minimal essential medium, DMEM-F12 growth medium, and McCoy's 5A medium mixed with $10.0 \%$ fetal bovine serum (FBS) that were purchased from Gibco (Gibco, USA). The cells were incubated overnight at $37^{\circ} \mathrm{C}$ in a $5 \% \mathrm{CO} 2$ humidified environment. The cells were trypsinized when they reached $75 \%$ confluence and were then used for in vitro and in vivo studies.

\section{Quantitative Real-Time Reverse Transcriptase-Polymerase Chain Reaction}

Quantitative real-time RT-PCR was performed in triplicate with an Applied Biosystems Prism 7,500 Fast Sequence Detection System using TaqMan universal PCR master mix according to the manufacture's protocol (Applied Biosystems Inc., Foster City, California, USA). TaqMan probes and primers were purchased from Applied Biosystems Inc. Levels of RNA expression were determined using the 7,500 Fast System SDS software package (version 1.3.1; Applied Biosystems Inc.) and 
GAPDH (glyceraldehyde 3-phosphate dehydrogenase) was used as a control for normalization. Primers used here were as follows: GAPDH for: 5'-TGACTTCAACAGCGACAC- -CCA3',GAPDH, reverse:5'-CACCCTGTTGCTGTAGCCAAA-3', DGKZ for: 5'-AGCAAG-CAAGAAGAAGAAGAGG-3', and DGKZ reverse:5'-GGATTGAGATACCAGAGGAAAGAC-3'. The relative DGKZ expression was normalized to GAPDH, and data analysis was conducted using the comparative CT method.

\section{DGKZ shRNA Design and Lentivirus Construction}

Targeted shRNA was used to knockdown the expression of DGKZ in OS cells. Sets of lentiviral plasmids pGCSILGFP (GeneChem, Shanghai, China) with sequences targeting to DGKZ mRNA(sense, 5'-TCGCACAGGATG AGATTTATA- $3^{\prime}$; antisense, $5^{\prime}$-TATAAATCTCATC- CTG TGCGA-3') and pGCSIL-GFP with non-targeting sequence as negative control were used for gene knockdown studies. Infectious shRNA-lentiviruses were packaged in $293 \mathrm{~T}$ cells, purified and used to infect U2OS, Saos2 and HOS cells for generation of stable transduced clones.

\section{Cell Proliferation Analysis}

Cells in shCtrl and shDGKZ groups were plated in 96-well plates at a density of $2 \times 10^{3}$ cells/well and incubated for 1-5 days. Each group contained three wells. At the end of incubation, 10 $\mu \mathrm{l}$ of $5 \mathrm{mg} / \mathrm{mL}$ MTT (Genview, USA) was added and incubated for $4 \mathrm{~h}$ at $37^{\circ} \mathrm{C}$. The medium was removed and $150 \mu \mathrm{L}$ DMSO was added. Absorbance was determined with an enzyme-linked immunosorbent assay reader at $595 \mathrm{~nm}$. The cell proliferation curves were drawn according to the absorbance.

Cell proliferation was also recorded and accessed through counting viable cell number with Cellomics Array-ScanTM VTI HCS Reader (Thermo Scientific, Waltham, MA, USA)by manufacturer's instructions. Briefly speaking, cells were cultured at a density of $2^{*} 10^{3} /$ well in 96 -well plates under $37^{\circ} \mathrm{C}$ with $5 \%$ $\mathrm{CO}_{2}$. From the next day, cells with GFP were taken photos and counted each day. Cell proliferation was recorded consecutively for 5 days. Cell growth curves were drawn according to cell numbers.

\section{Cell Apoptosis Analysis}

Cell apoptosis was assayed by staining with Annexin V-APC and detected by flowcytometry. Briefly, cells were washed twice with cold PBS and resuspended in $1 \times$ binding buffer. Then $100 \mu \mathrm{l}$ of solution (about $1 \times 10^{6}-1 \times 10^{7}$ cells) was transferred to a

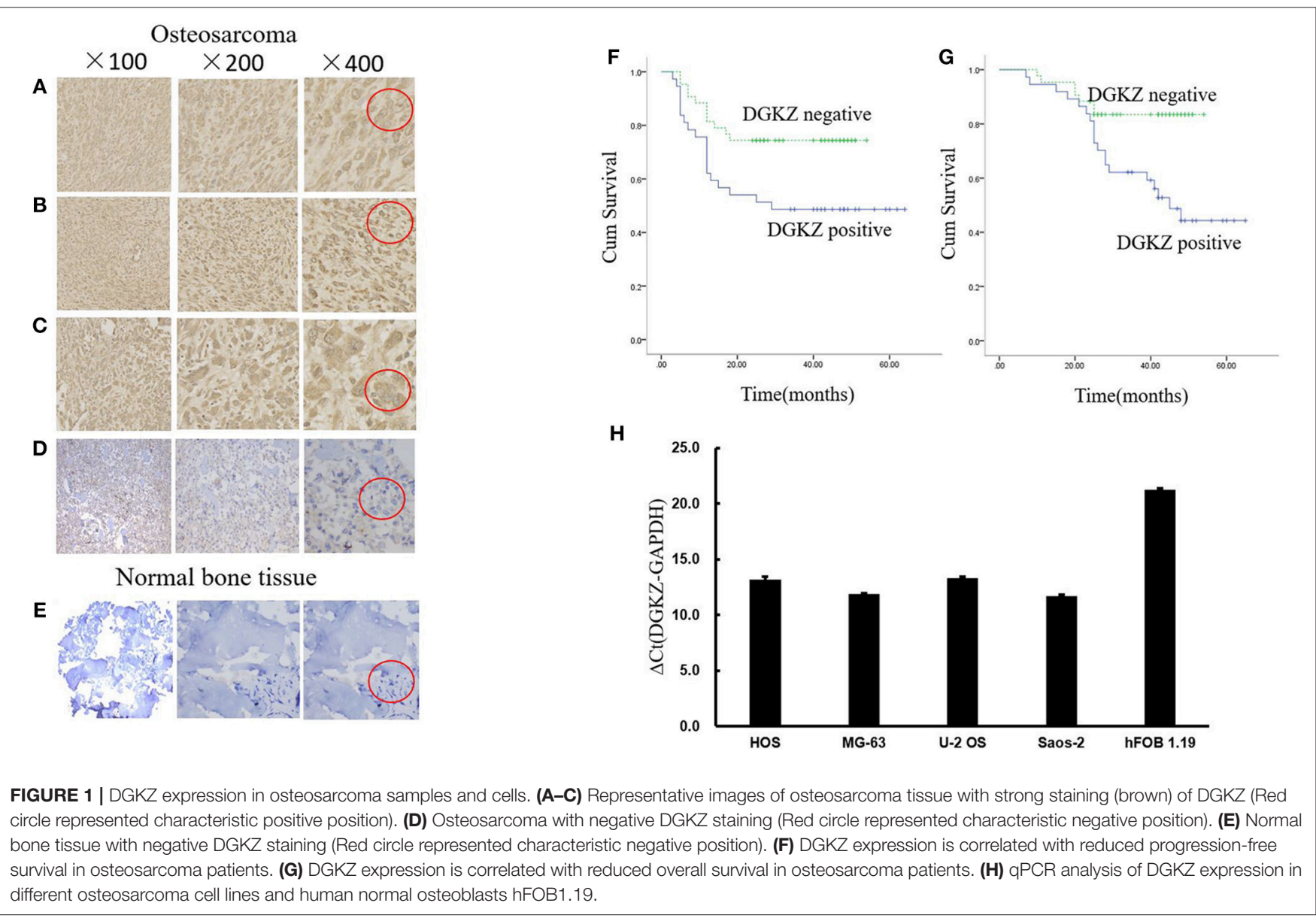


$5 \mu \mathrm{l}$ Annexin V-APC and $5 \mu \mathrm{l}$ PI, and incubated for $15 \mathrm{~min}$ at room temperature in the dark. Cells were analyzed using flow cytometry. All experiments were performed in triplicate.

The activity of Caspases3/7 in OS cells was detected with Caspase-3/7 Assay Kit (Promega), following the manufacturer's instructions and cell fluorescence intensity at $499 \mathrm{~nm}$ was measured by ELISA Tablet counter for quantitative assessment.

\section{Assessment of Tumor Growth Inhibitory Effects of DGKZ -Silencing in a Xenograft Model}

The mouse experiments and animal care procedures were approved by the Ethics Committee of Affiliated Sixth People's Hospital, Shanghai Jiaotong University. Four-week-old male $\mathrm{BALB} / \mathrm{c}$ nude mice were obtained from Shanghai Laboratory Animal Center (Shanghai, China). HOS cells $\left(4 \times 10^{6}\right)$ stably expressing DGKZ or shDGKZ were suspended in $150 \mu \mathrm{L}$ of phosphate-buffered saline and inoculated subcutaneously in the right armpit region ( $n=10$ per group). After 4 weeks, the mice were sacrificed and the tumors were removed for analysis. Tumor volumes were calculated using the following formula six times during observation by Vernier calipers: 3.14/6 $\times$ y (length) $\times$ $\mathrm{x}^{2}$ (width). After the mice were killed, the tumors were resected and weighted. In addition, before sacrificed, tumor growth in the living animals was monitored and quantified by luminescence levels. Bioluminescence was measured with the IVIS imaging system (Xenogen Corp., Alameda, CA, USA). All of the images were taken $10 \mathrm{~min}$ after intraperitoneal injection of luciferin (Sigma-Aldrich, St. Louis, MO, USA) of $150 \mathrm{mg} / \mathrm{kg}$ body weight, as a 60 -s acquisition and 10 of binning. During image acquisition, mice were sedated continuously via inhalation of $3 \%$ isoflurane. Image analysis and bioluminescent quantification was performed using Living Image software (Xenogen Corp).

TABLE 1 | Characteristics of 80 osteosarcoma patients.

\begin{tabular}{lcc}
\hline & DGKZ -positive & DGKZ -negative \\
\hline GENDER & 26 & 29 \\
Male & 11 & 14 \\
Female & & \\
AGE & 22 & 25 \\
$\geq 18$ years & 15 & 18 \\
$<18$ years & & \\
HISTOTYPE OF TUMOR & 33 & 38 \\
Conventional & 4 & 5 \\
Other & 26 & 30 \\
INITIAL TUMOR SITE & 3 & 5 \\
Femur & 7 & 5 \\
Tibia & 1 & 3 \\
Humerus & & 9 \\
Fibula & 11 & 34 \\
METHOD OF SURGERY & 26 & \\
Amputation & & \\
Limb salvage & &
\end{tabular}

All factors were well balanced between two groups $(p>0.05)$.

\section{Microarray Gene Expression Analysis}

After infection with shDGKZ total RNA was extracted from HOS osteosarcoma cells, and 50-500 ng of RNA was used to generate biotin-modified amplified RNA (aRNA) using a GeneChip primeview human Kit (Affymetrix, USA). Data of gene expression was analyzed by GeneChip ${ }^{\circledR}$ PrimeView ${ }^{\text {TM }}$ Human Gene Expression Array (Affymetrix, USA; catalog \# 901838) which contains probes for 36,000 genes and subsequent analysis was performed using the Affymetrix Expression Console (EC, version 1.1).For the microarray analysis, following filtering by flag signal, the raw data were processed and normalized. Differentially expressed genes were identified with $P<0.05$ and fold changes $>2$. In addition, IPA (Ingenuity Pathway Analysis) was performed which provided more detailed information regarding the pathways in which the target genes were involved.

\section{Affinity Purification of DGKZ Interacting Complexes and LC-MS/MS Analysis}

The DGKZ gene fused with a $3 \times$ FLAG tag sequence (Sigma) at its $5^{\prime}$-end was amplified by PCR and inserted into GV367 lentiviral vector (Genechem, Shanghai, China), and the construct was named as p3 $\times$ FLAG-DGKZ. HOS cells were plated at the concentration of $4 \times 10^{5}$ cells/well in 6-well plate, $2 \mathrm{ml}$ of DMEM medium containing $10 \mu \mathrm{l}$ of $3 \times$ FLAG-DGKZ or negative control lentivirus and $2 \mu \mathrm{l}$ of polybrene $(10 \mu \mathrm{g} / \mu \mathrm{l})$ was added into the wells. The stable cells expressing $3 \times$ FLAG-DGKZ was validated by immunoblotting using the specific antibodies. The $3 \times$ FLAGDGKZ interacting complex was purified using the anti-FLAG magnetic beads (Sigma) according to the manufacture's protocol. The procedure of in-gel trypsin digestion and peptide extraction was performed following the standard protocol. LC-MS analysis was performed on a Nano Acquity UPLC system (Waters Corporation, MA, U.S.) connected to a quadrupole-Orbitrap mass spectrometer (Q-Exactive) (Thermo Fisher Scientific, Bremen, Germany) equipped with an online nano-electrospray ion source. The Q-Exactive mass spectrometer was operated in the data-dependent mode to switch automatically between MS and MS/MS acquisition. Data from both scans were acquired in profile mode. The spectra were recorded with Xcalibursoftware.

The raw mass files generated by the Q-Exactive instrument were processed using Proteome Discoverer software (Thermo Scientific, version 1.4) integrated with the MASCOT (Matrix Science, London, UK; version 2.3.2) search engine for protein identification. Data were searched against the Human UniProtKB/Swiss-Prot database (Release 2017_02_19, 20185 entries).To reduce false positive identification results, a decoy database containing the reverse sequences was appended to the database.

\section{Co-immunoprecipitation and Immunoblotting}

The procedure of protein isolation and immunoprecipitation was essentially the same as described in the purification of $3 \times$ FLAG-DGKZ complexes for MS identification. The immunoprecipitated protein complexes were eluted by $3 \times$ FLAG peptide. Samples were separated by SDS-PAGE and 
transferred on PVDF membrane (Millipore, $0.2 \mu \mathrm{m}$ ). The membranes were blocked with 5\% non-fat milk and probed with specified primary antibody followed by incubation with the secondary antibody conjugated with horseradishperoxidase, and then the ECL substrate was added on membrane and exposed by ECL system.

\section{Statistical Analysis}

Patient survival was analyzed by the log-rank test using SPSS 15.0 software. All experiments were performed in triplicates, and the data were expressed as the mean \pm SD. Statistical analyses were conducted with Student's $t$-test. $P<0.05$ was considered statistically significant.
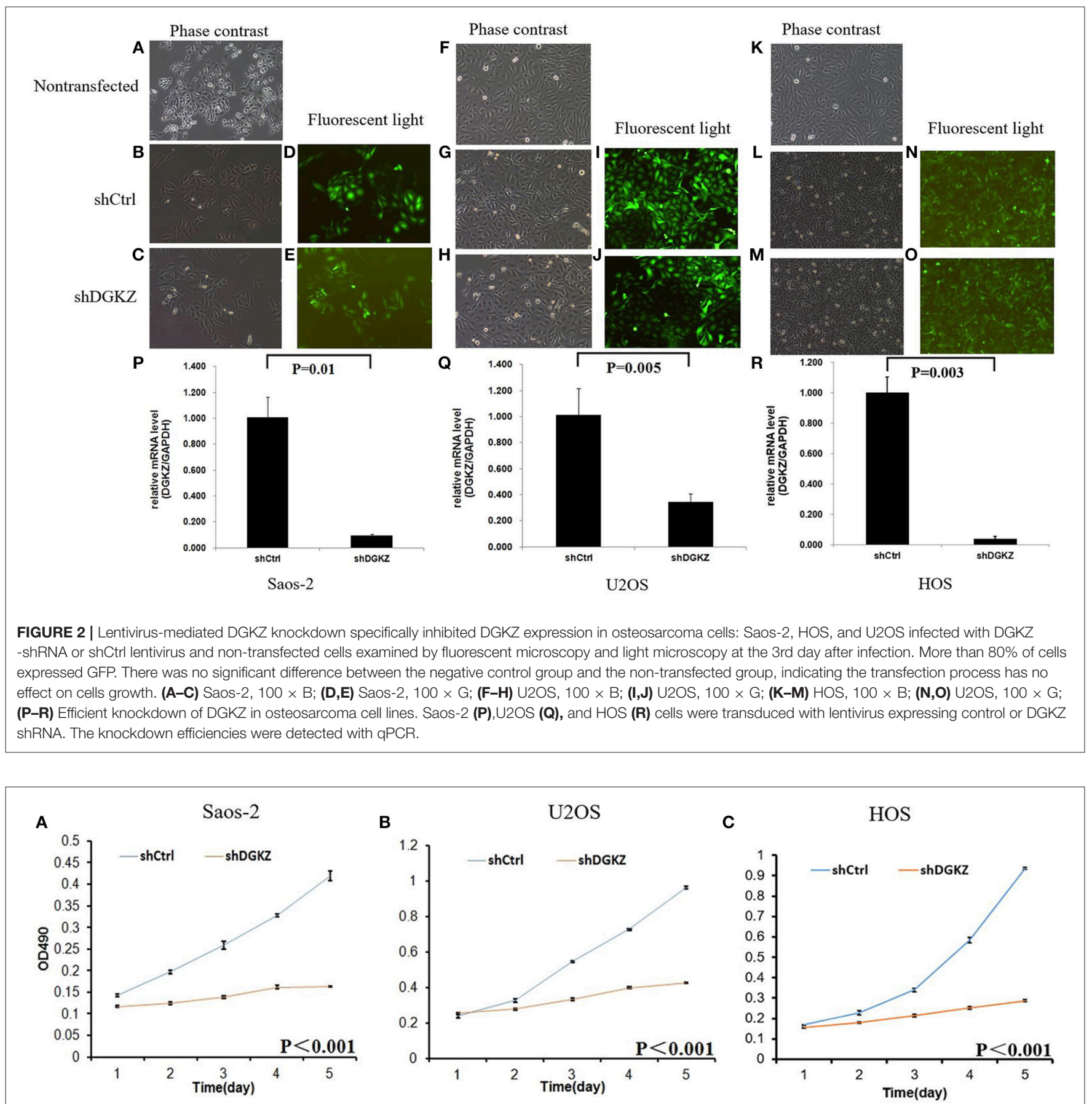

FIGURE 3 | Knockdown of DGKZ inhibited cell proliferation ability in osteosarcoma cells by MTT assay: Saos-2 (A), U2OS (B), and HOS(C). 


\section{RESULTS}

\section{High Expression of DGKZ in Osteosarcoma Samples/Cells and Its Correlation With Poor Prognosis}

To initially detect expression of DGKZ in osteosarcoma, we collected 80 osteosarcoma samples and 24 normal bone samples. Among 80 tumor samples, 37 osteosarcoma samples(46.2\%) had high expression of DGKZ with a score defined as positive (above 6). Compared with osteosarcoma samples, DGKZ expression was absent in normal bone samples while all bone samples demonstrated a score defined as negative $(\leq 6$, Figures 1A-E). Additionally, DGKZ was detected to distribute in cytoplasm. Furthermore, The level of DGKZ expressed in four OS cell lines (SaoS-2, U-2OS, HOS, MG-63) was significantly higher than normal osteoblasts hFOB1.19 (Figure 1H).

No correlation was observed between age/gender/tumor location/pathological subtype and DGKZ expression (Table 1). DGKZ expression was associated with a poor prognosis in IIB limb osteosarcoma patients. According to data from follow-up, while evaluating progression-free survival, patients with DGKZ positive expression had a mean survival time of 36.7 months, whereas patients whose samples with DGKZ negative expression had a mean survival time of 42.9 months $(P=0.018$, Figure 1F).
Additionally, DGKZ expression was also associated with poor overall survival [mean, 44.7 months (positive) vs. 48.2 months (negative), $P=0.007$, Figure 1G].

In summary, these results demonstrated that OS patients with high DGKZ expression survived for shorter periods than those with low DGKZ expression and DGKZ might played an essential role in the development and progression of osteosarcoma.

\section{DGKZ Silencing Inhibited Growth and Promoted Apoptosis in vitro}

According to the above result, we further explored DGKZ biological function through RNA interference. We did Lentivirus-mediated knockdown of DGKZ in Saos-2, U2OS and HOS cells. Fluorescent microscopy and light microscopy demonstrated that more than $80 \%$ of cells expressed GFP at the 3rd day after infection (Figures 2A-O). Compared with mock transfected cells, DGKZ's expression was significantly inhibited in cells with shRNA at mRNA level by qPCR analysis, which demonstrated that the lentiviral-based shRNA construct exerted a specific knockdown effect on endogenous DGKZ expression in Saos-2, U2OS, and HOS cells (Figures 2P-R).

Sustaining proliferation signaling is one of the core hallmarks of cancer (33). Thus, we investigated cell proliferation in Saos2, U2OS, and HOS cells with or without shDGKZ by HCS and

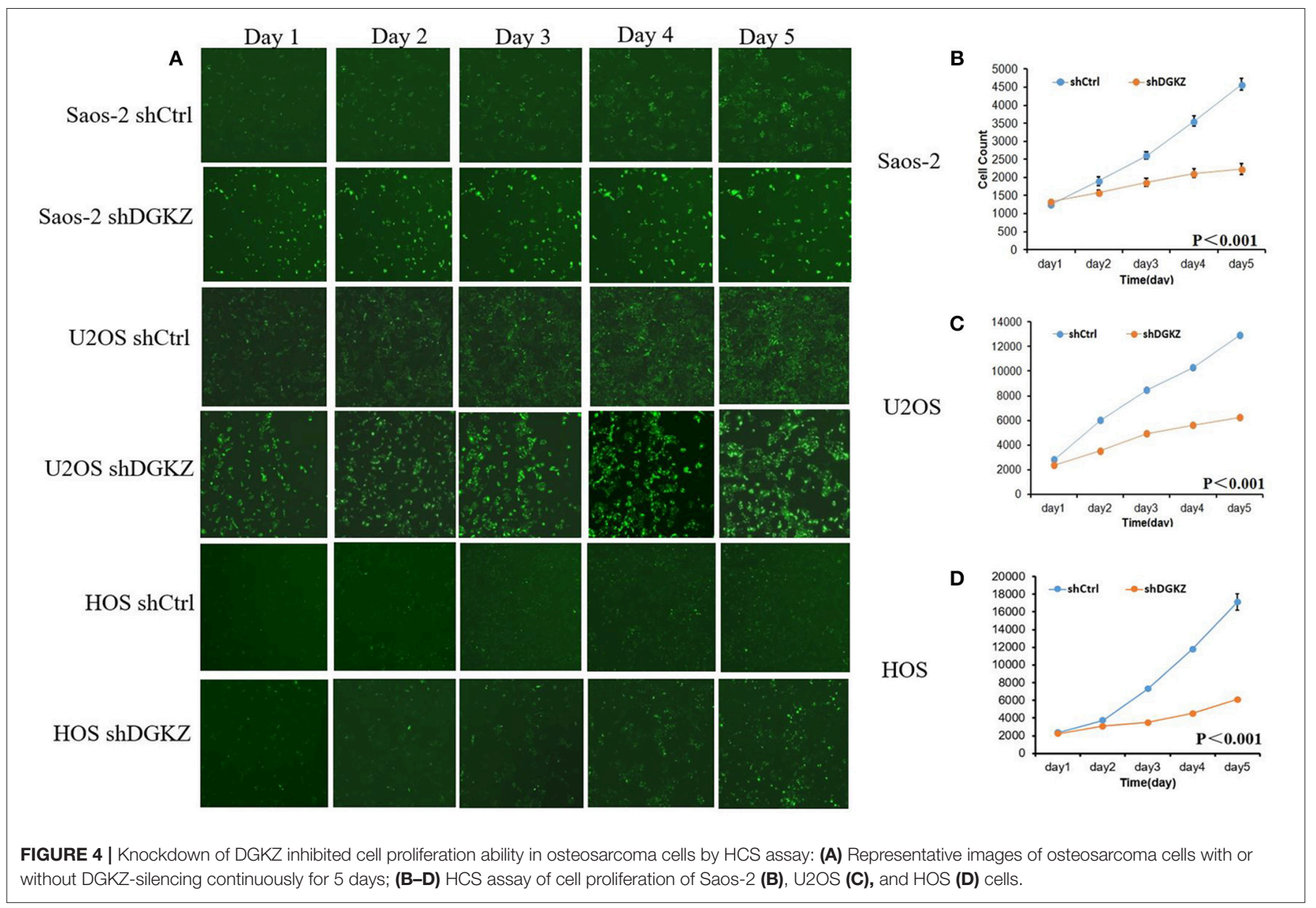


MTT assays. As shown in Figures 3, 4, significant decrease in proliferation was observed in shDGKZ group by MTT and HCS assay in all three cell lines.

Resisting cell death, another hallmark of cancers, is essential for tumors (34). Thus, we evaluated Caspase3/7 activity in Saos-2, U2OS, and HOS cells with shDGKZ. As demonstrated in Figures 5A-C, Caspase3/7 activity, measured by using a luminescent caspase activity assay kit, increased remarkably in DGKZ-silencing Saos-2/U2OS/HOS cells compared with control group. Annexin V-APC staining by FACS in Saos-2,U2OS and HOS cells following DGKZ -knockdown was also administrated to check impact of GINS1 on apoptosis. Figures 5D-F showed the apoptotic rate in DGKZ-silencing cells was much higher than that in control group in all three cell lines, suggesting that DGKZ knockdown facilitated apoptosis of osteosarcoma cells.

\section{Silencing of DGKZ Inhibits Tumorigenicity in vivo}

We next examined whether DGKZ silencing in osteosarcoma affects OS cell growth in vivo. Right armpit region of $\mathrm{BALB} / \mathrm{c}$ nude mice was chosen to perform subcutaneous injection with HOS cells with or without DGKZ-knockout. Volume of tumor was checked continuously after cell implantation under overall health status for all individuals. Tumors were removed and then subjected to measure weight. Consistent with our in vitro findings, DGKZ knockdown impaired proliferation
(Figures 6A,B). Meanwhile, through bioluminescence measurement, as shown in the Figures $6 \mathrm{C}-\mathrm{E}$, the control group showed steeper progression compared with the DGKZsilenced group. After scarification, the averaged tumor weight of the control mice was extremely heavier than that in DGKZsilenced group which presented suppressed tumor growth (Figures 6F,G). These results indicated that knockout of DGKZ inhibited tumor growth of osteosarcoma in vivo.

\section{Gene Expression Profile Analysis Revealed MYC Pathway Was a Downstream Target Affected by DGKZ}

The previous data suggested that DGKZ played a key role in osteosarcoma growth. However, mechanisms underlying DGKZ-regulation are still unclear. Thus, Affymetrix GeneChip and IPA were administrated to display an overview of DGKZ possible biological interaction through DGKZ knockdown in HOS cells. Differentially expressed genes with at least a 1.5-fold change were identified. As shown in Figure 7A, 807 genes were up-regulated and 1,110 genes were down-regulated in DGKZ knockdown HOS cells compared with negative control cells. Ingenuity Pathway Analysis (IPA) revealed that several functional classifications were significantly enriched, including cell assembly/organization, cell death/survival, organismal survival, cell cycle, cellular function/maintenance, cellular development, cellular growth/proliferation, cellular movement,

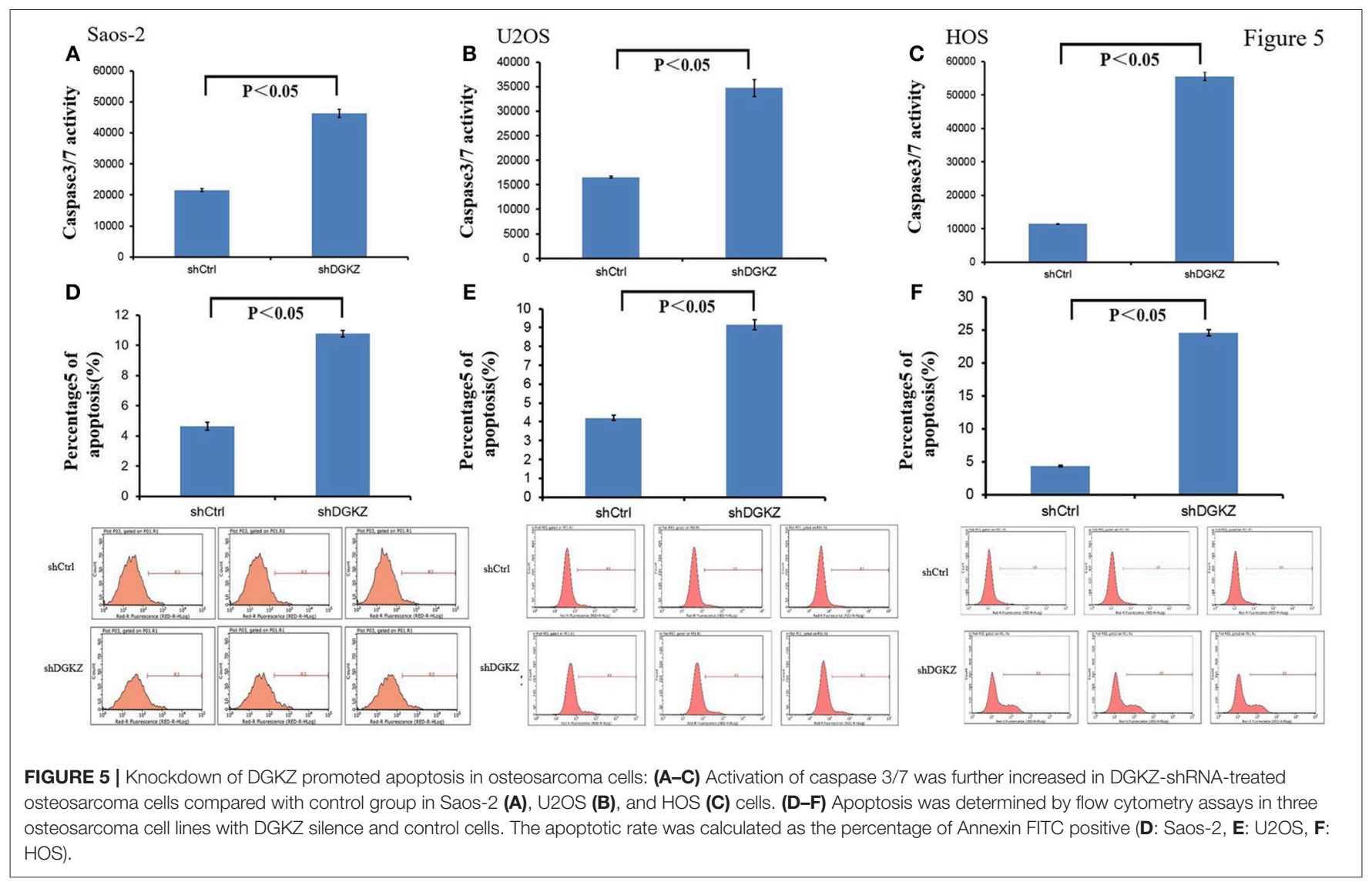




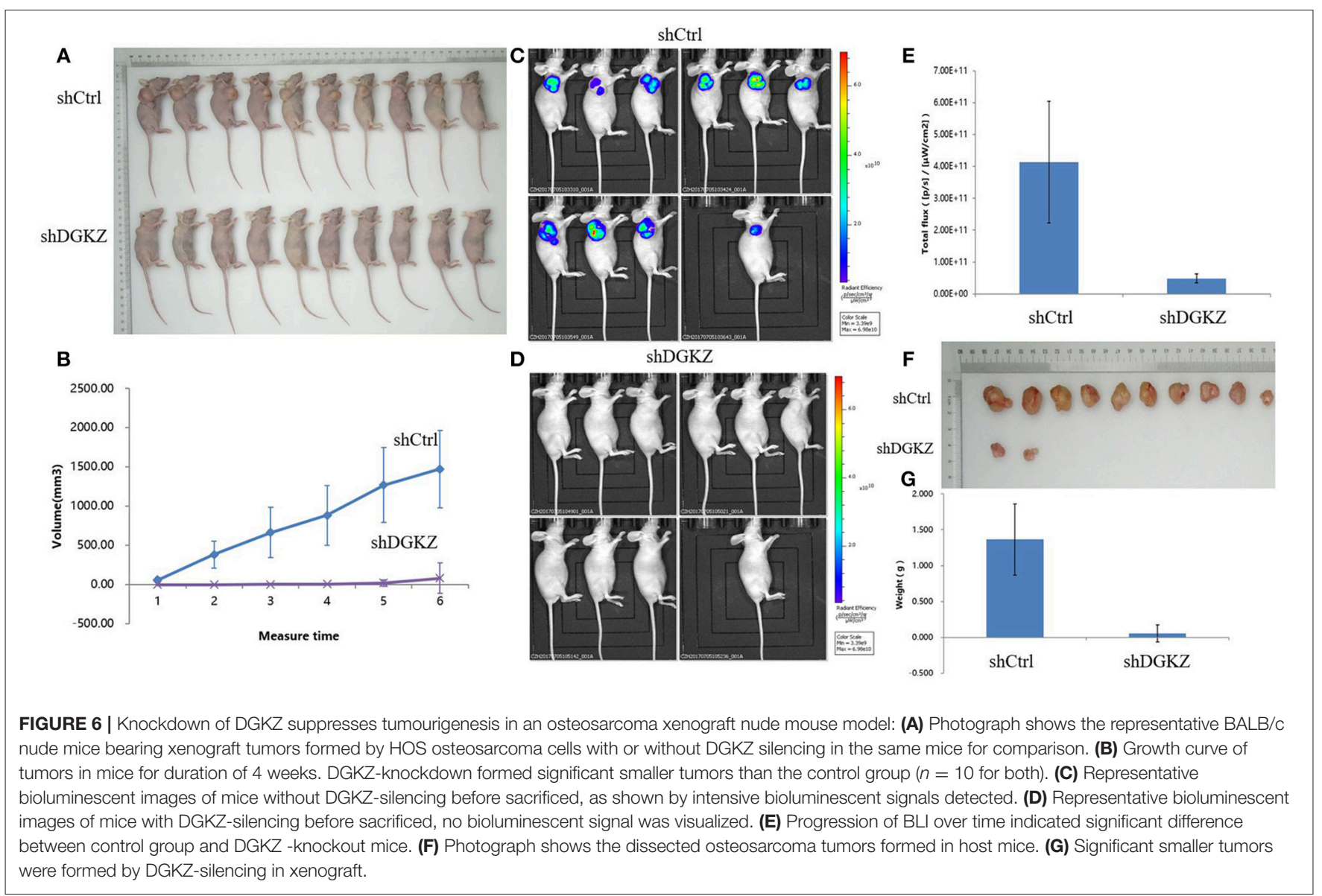

tumor morphology, and cell signaling (Supplemental Figure 1). Further, IPA in "canonical pathway" module revealed that several critical pathways involved in cancer development and apoptosis such as "ILK signaling," "signaling by Rho Family GTPases," and "BMP signaling pathway" were activated while pathways such as "RhoGDI signaling" was inhibited by DGKZ knockdown(Supplemental Figure 2). These data demonstrated that the effects of DGKZ in osteosarcoma progression were likely dependent on regulation of several key cellular functions.

Additionally, selected gene lists obtained from the microarray analyses were uploaded to IPA system and a core biologic pathway analysis was performed to identify molecular networks. Ingenuity Pathway Analysis (IPA) revealed that after DGKZ knockdown, target genes expression in MYC pathway were altered(Supplemental Figure 3), including CCND1, CDKN2B, CDK6, MYC, PCNA, and EGR1, which were further confirmed by Western blot (Figure 7B). Taken together, these data imply that role of DGKZ in osteosarcoma pathogenesis may function via regulation of MYC signal pathway.

\section{Identification of the ERK1/2 as DGKZ Interacting Proteins in MNNG/HOS Cells}

Co-immunoprecipitated proteins were separated using SDSPAGE and stained (Figure 7C). Coomassie staining of the gels loaded with DGKZ-IP identified several bands that were not present in vector or IgG control. Immunoprecipitated proteins were gel extracted, trypsin digested, and identified by LC-MS/MS analysis (Figure 7D). We identified 100 unique proteins with a protein False Discovery Rate equal or lower than 1\%. We then applied a manual thresholding approach and a probabilistic PPI prediction algorithm to compute the most likely associations between each of these 100 proteins and DGKZ. Followed by co-immunoprecipitation and immunoblotting, we identified ERK1/2, a key component in Ras/Raf/MAPK pathway and a key MYC-interactor, as a candidate protein interacted with DGKZ in HOS cells (Figure 7E).

\section{DISCUSSION}

Osteosarcoma is the most common type of primary malignant bone tumor affecting both children and adults (35). Even the outcome for patients with osteosarcoma has improved dramatically by therapeutic strategies, those who suffered from distant metastasis or local recurrence continue to pose a particularly tough challenge (3). Thus, a better understanding of the molecular biology of OS is needed and may improve therapeutic efficiency. Diacylglycerol kinase zeta (DGKZ) has proven to be involved in several physiological or pathophysiological signaling pathways, including certain human 
A

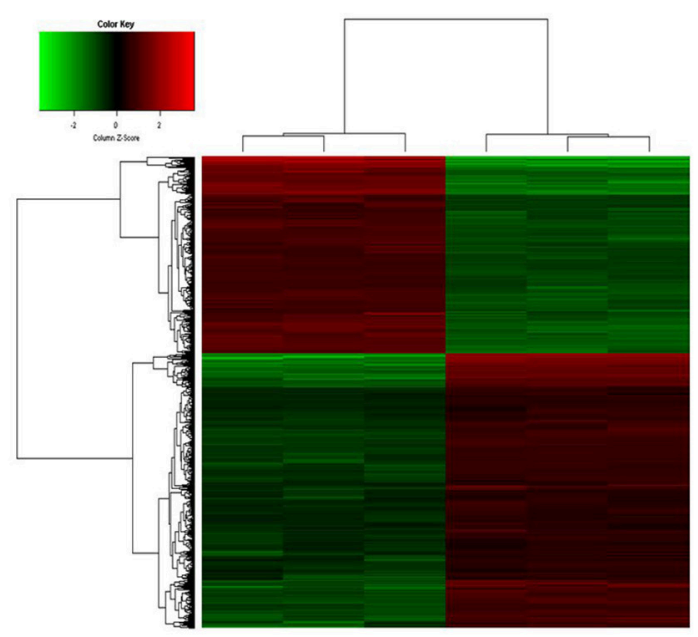

shDGKZ
B
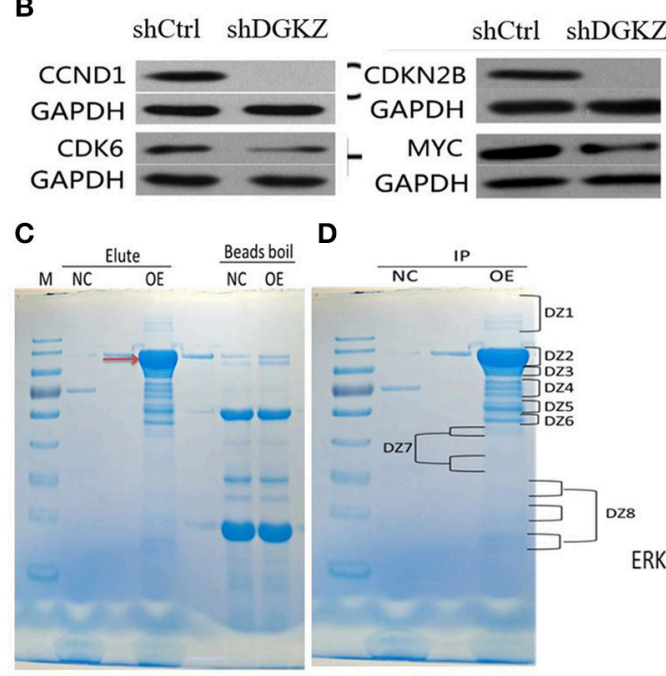

D

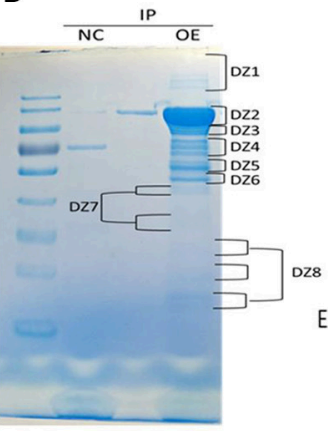

E
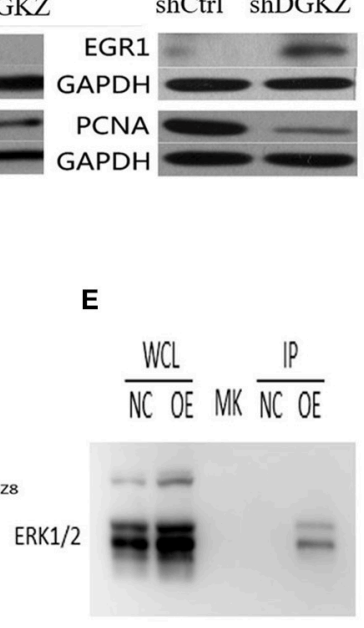

FIGURE 7 | Effects of DGKZ knockdown on downstream gene expression according to microarray assay and identification of DGKZ's interaction protein through LC-MS/MS analysis: (A) Heatmap representation of genes significant differential expressions in HOS cells infected with lentivirus expressing shCtrl or shDGKZ under the criteria $p<0.05$ and $\mid$ fold change $\mid>1.5$. Genes and samples were listed in rows and columns, respectively. A color scale for the normalized expression data was shown at the bottom of the microarray heatmap (green represents downregulated genes while red represents upregulated genes). (B) Validation of downstream gene target expression in MYC pathway by Western blot. (C) The $3 \times$ FLAG-DGKZ interacting complex was purified using the anti-FLAG magnetic beads (Red arrow: $3 \times$ FLAG-DGKZ). In contrast to the control (sample pulled-down from control cells), the bands only in sample pulled-down from $3 \times$ FLAG-DGKZ cells were excised for the following in-gel trypsin digestion(NC: negative control, OE: over expression). (D) The gel band was cut into pieces and washed with Milli-Q water. The tryptic peptides were extracted from the gel pieces and lyophilized by vacuum centrifugation. (E) Through LC-MS analysis and further co-immunoprecipitation/ immunoblotting, interaction between ERK1/2 and DGKZ was confirmed in HOS cells.

carcinogenesis. In our study, we speculated that DGKZ acts as an oncogene in proliferation of osteosarcoma. Expression of DGKZ was significantly up-regulated in OS tumor samples compared with normal bone tissue, and was associated with poor prognosis. Moreover, in vitro studies, through a constructed lentivirus expressing DGKZ-specific shRNA, we further demonstrated the key role of DGKZ in cell growth and apoptosis in Saos2/U2OS/HOS cells. Additionally, considering that a suitable animal model was needed to investigate the effect of DGKZ in vivo, we chose BALB/c nude mice, one of the most widely used models, to establish xenograft model induced by subcutaneous injection of HOS cells. Consistent with findings in vitro, our data also strongly supported the positive correlation between DGKZ expression and osteosarcoma growth. Taken together, these data indicated substantial evidence that DGKZ is a prognostic marker and potential new target for cancer therapy in osteosarcoma.

Previous data demonstrated that DGKZ was involved in tumor initiation and progression (26-28). Cai et al. (27) found that high DGKZ expression could facilitate Rho GTPase activation and promote motility of metastatic function in colon cancer. Diao et al. (28) demonstrated positive correlation between DGKZ expression and gliomagrade in glioma tumor samples. Furthermore, DGKZ knockdown in human glioma suppressed cell proliferation, hampered colony formation ability, and promoted cell cycle arrest and apoptosis. Torres-Ayuso et al. (26) explored the potential molecular mechanism of DGKZ's role in rapamycin-resistant colon cancer cell line, and found that DGKZ manipulated mTORC1 and lipogenic metabolism in colon cancer cells through SREBP-1. In line with these data, we also proved that role of $\mathrm{DGKZ}$ acts an oncogene in proliferation of osteosarcoma.

The regulation of DGKZ on downstream signal pathway in cancer cells is still not well-elucidated. To investigate the potential mechanism about DGKZ in regulating tumorigenesis of osteosarcoma, we conducted microarray analysis to explore alteration of cancer-related genes between normal osteosarcoma cells and DGKZ knockdown cells. Microarray data demonstrated that DGKZ's oncogene role is the result of regulating a number of different signaling pathways, which is consistent with a biofunctional role for DGKZ reported in previous study $(16,18-25)$. IPA analysis with confirmed western blot data demonstrated that DGKZ siRNA-expressing cells posed a downregulated activity of MYC signal pathway and an altered expression of several target genes in MYC pathway. MYC proto-oncogene promotes oncogenic transcriptional amplification program in cancers and represents as an important therapeutic target for cancer therapy. In addition, misregulated expressions of MYC are often associated with osteosarcoma oncogenesis and progression (36). Taken together, it could be concluded that DGKZ might exert an enhancing effect on activity of MYC signal pathway to impact on osteosarcoma proliferation. 
The MYC signaling pathway consists of a family of upstream and downstream targets that are associated with cell proliferation and growth. Whether regulation of DGKZ on downstream MYC pathway in osteosarcoma is through a direct or indirect route is still not clear. In our study, through IP-MS analysis, we provided potential evidence that the interaction of ERK1/2 and DGKZ was closely linked to MYC-dependent osteosarcoma development. ERK1/2 are widely expressed in various cells including cardiomyocytes, neurons, and hepatocytes. They are directly activated by phosphorylation of mitogen-activated protein kinase (MAPK) to promote cellular differentiation, proliferation, and survival (37). In pathological situation, such enhanced/constitutive activation of ERK1/2 existed in various human malignancies, including osteosarcoma (38-40). Various articles have showed activation of the ERK-pathway prolonged the half-life of the MYC protein and thus enhances the accumulation of MYC activity, both in normal cell growth control and oncogenesis $(41,42)$. This finding enriched our knowledge on how DGKZ exerted an impact in osteosarcoma MYC-dependent proliferation. Thus, we speculated that DGKZ might regulate MYC signal pathway via possible interaction with ERK1/2 in carcinogenesis of osteosarcoma and utilization of DGKZ-ERK1/2-MYC interacting axis inhibitor might be an effective approach in treatment of osteosarcoma. However, the detailed mechanism of MYC pathway regulation induced by DGKZ-ERK1/2 needs further investigation.

In conclusion, our study demonstrated that DGKZ, a potential prognostic and predictive indicator in osteosarcoma, play a key role in proliferation of osteosarcoma, via its possible interaction with ERK1/2 and regulation of MYC pathway. These data provide an important starting point for future studies investigating the potential of DGKZ-ERK1/2-MYC axis as a potential new target for cancer therapy in osteosarcoma.

\section{AUTHOR CONTRIBUTIONS}

WY drafted the manuscript, participated in the design of the study, data collection. and analysis. LT and FL participated in data collection and analysis. YY was involved in drafting the manuscript. ZS designed the study and helped to draft the manuscript. All authors read and approved the final manuscript.

\section{FUNDING}

This study was supported by NSFC 81502325 and 81672658 (NSFC: National Natural Science Foundation of China).

\section{SUPPLEMENTARY MATERIAL}

The Supplementary Material for this article can be found online at: https://www.frontiersin.org/articles/10.3389/fonc. 2018.00655/full\#supplementary-material

Supplemental Figure 1 | DGKZ related disease and function enrichment was analyzed based on IPA databases.

Supplemental Figure 2 | DGKZ related functional pathway enrichment was analyzed based on IPA databases.

Supplemental Figure 3 | Up-stream and down-stream network of MYC analyzed by IPA.

\section{REFERENCES}

1. Mirabello L, Troisi RJ, Savage SA. Osteosarcoma incidence and survival rates from 1973 to 2004: data from the Surveillance, Epidemiology, and End Results Program. Cancer (2009) 115:1531-43. doi: 10.1002/cncr.24121

2. Luetke A, Meyers PA, Lewis I, Juergens H. Osteosarcoma treatment where do we stand? A state of the art review. Cancer Treat Rev. (2014) 40:523-32. doi: 10.1016/j.ctrv.2013.11.006

3. Isakoff MS, Bielack SS, Meltzer P, Gorlick R. Osteosarcoma: current treatment and a collaborative pathway to success. J Clin Oncol. (2015) 33:3029-35. doi: 10.1200/JCO.2014.59.4895

4. Huang J, Ni J, Liu K, Yu Y, Xie M, Kang R, et al. HMGB1 promotes drug resistance in osteosarcoma. Cancer Res. (2012) 72:230-8. doi: 10.1158/0008-5472.CAN-11-2001

5. Li S, Sun W, Wang H, Zuo D, Hua Y, Cai Z. Research progress on the multidrug resistance mechanisms of osteosarcoma chemotherapy and reversal. Tumour Biol. (2015) 36:1329-38. doi: 10.1007/s13277-015-3181-0

6. Bielack SS, Kempf-Bielack B, Delling G, Exner GU, Flege S, Helmke K, et al. Prognostic factors in high-grade osteosarcoma of the extremities or trunk: an analysis of 1,702 patients treated on neoadjuvant cooperative osteosarcoma study group protocols. J Clin Oncol. (2002) 20:776-90. doi: 10.1200/JCO.2002.20.3.776

7. Chen X, Bahrami A, Pappo A, Easton J, Dalton J, Hedlund E, et al. Recurrent somatic structural variations contribute to tumorigenesis in pediatric osteosarcoma. Cell Rep. (2014) 7:104-12. doi: 10.1016/j.celrep.2014.03.003

8. Cockcroft S, Thomas GM. Inositol-lipid-specific phospholipase C isoenzymes and their differential regulation by receptors. Biochem J. (1992) 288(Pt 1):114. doi: 10.1042/bj2880001

9. Divecha N, Irvine RF. Phospholipid signaling. Cell (1995) 80:269-78. doi: 10.1016/0092-8674(95)90409-3

10. Topham MK. Signaling roles of diacylglycerol kinases. J Cell Biochem. (2006) 97:474-84. doi: 10.1002/jcb.20704

11. Kanoh H, Yamada K, Sakane F. Diacylglycerol kinase: a key modulator of signal transduction? Trends Biochem Sci. (1990) 15:47-50. doi: 10.1016/0968-0004(90)90172-8

12. Topham MK, Epand RM. Mammalian diacylglycerol kinases: molecular interactions and biological functions of selected isoforms. Biochim Biophys Acta. (2009) 1790:416-24. doi: 10.1016/j.bbagen.2009.01.010

13. Sakane F, Imai S, Kai M, Yasuda S, Kanoh H. Diacylglycerol kinases: why so many of them? Biochim Biophys Acta (2007) 1771:793-806. doi: 10.1016/j.bbalip.2007.04.006

14. Mérida I, Avila-Flores A, Merino E. Diacylglycerol kinases: at the hub of cell signaling. Biochem J. (2008) 409:1-18. doi: 10.1042/BJ20071040

15. Goto K, Hozumi Y, Nakano T, Saino SS, Kondo H. Cell biology and pathophysiology of the diacylglycerol kinase family: morphological aspects in tissues and organs. Int Rev Cytol. (2007) 264:25-63. doi: 10.1016/S0074-7696(07)64002-9

16. Hozumi Y, Ito T, Nakano T, Nakagawa T, Aoyagi M, Kondo H, et al. Nuclear localization of diacylglycerol kinase zeta in neurons. Eur J Neurosci. (2003) 18:1448-57. doi: 10.1046/j.1460-9568.2003.02871.x

17. Bunting M1, Tang W, Zimmerman GA, McIntyre TM, Prescott SM. Molecular cloning and characterization of a novel human diacylglycerol kinase zeta. J Biol Chem. (1996) 271:10230-6. doi: 10.1074/jbc.271.17. 10230

18. Rincón E, Gharbi SI, Santos-Mendoza T, Mérida I. Diacylglycerol kinase $\zeta$ : at the crossroads of lipid signaling and protein complex organization. Prog Lipid Res. (2012) 51:1-10. doi: 10.1016/j.plipres.2011.10.001

19. Ard R, Mulatz K, Pomoransky JL, Parks RJ, Trinkle-Mulcahy L, Bell JC, et al. Regulation of Macropinocytosis by Diacylglycerol Kinase $\zeta$. PLoS ONE (2015) 10:e144942. doi: 10.1371/journal.pone.0144942 
20. Zamani A, Decker C, Cremasco V, Hughes L, Novack DV, Faccio R. Diacylglycerol Kinase $\zeta$ (DGKZ) Is a critical regulator of bone homeostasis via modulation of c-Fos levels in osteoclasts. J Bone Miner Res. (2015) 30:1852-63. doi: 10.1002/jbmr.2533

21. Hirose M, Takeishi Y, Niizeki T, Nakada T, Shimojo H, Kashihara T, et al. Diacylglycerol kinase $\zeta$ inhibits ventricular tachyarrhythmias in a mouse model of heart failure. Circ J. (2011) 75:2333-42. doi: 10.1253/circj.CJ-10-1213

22. Nakano T, Iseki K, Hozumi Y, Kawamae K, Wakabayashi I, Goto K. Brain trauma induces expression of diacylglycerol kinase zeta in microglia. Neurosci Lett. (2009) 461:110-5. doi: 10.1016/j.neulet.2009.06.001

23. Los AP, de Widt J, van Blitterswijk WJ, Divecha N. Is there a role for diacylglycerol kinase-zeta in cell cycle regulation? Adv Enzyme Regul. (2008) 48:31-9. doi: 10.1016/j.advenzreg.2008.02.001

24. Bilim O, Takeishi Y, Kitahara T, Arimoto T, Niizeki T, Sasaki T, et al. Diacylglycerol kinase zeta inhibits myocardial atrophy and restores cardiac dysfunction in streptozotocin-induced diabetes mellitus. Cardiovasc Diabetol. (2008) 7:2. doi: 10.1186/1475-2840-7-2

25. Nakano T, Hozumi Y, Ali H, Saino-Saito S, Kamii H, Sato S, et al. Diacylglycerol kinase zeta is involved in the process of cerebral infarction. Eur J Neurosci. (2006) 23:1427-35. doi: 10.1111/j.1460-9568.2006.04685.x

26. Torres-Ayuso P, Tello-Lafoz M, Mérida I, Ávila-Flores A. Diacylglycerol kinase- $\zeta$ regulates mTORC1 and lipogenic metabolism in cancer cells through SREBP-1.Oncogenesis (2015) 4:e164. doi: 10.1038/oncsis.2015.22

27. Cai K, Mulatz K, Ard R, Nguyen T, Gee SH. Increased diacylglycerol kinase $\zeta$ expression in human metastatic colon cancer cells augments Rho GTPase activity and contributes to enhanced invasion. BMC Cancer (2014) 14:208. doi: 10.1186/1471-2407-14-208

28. Diao J, Wu C, Zhang J, Liu J, Zhang X, Hao P, et al. Loss of Diacylglycerol Kinase-? inhibits cell proliferation and survival in human gliomas. Mol Neurobiol. (2016) 53:5425-35. doi: 10.1007/s12035-015-9419-4

29. Weekes D, Kashima TG, Zandueta C, Perurena N, Thomas DP, Sunters A, et al. Regulation of osteosarcoma cell lung metastasis by the c-Fos/AP-1 target FGFR1. Oncogene (2016) 35:2852-61. doi: 10.1038/onc.2015.344

30. Kakar S, Mihalov M, Chachlani NA, Ghosh L, Johnstone H. Correlation of c-fos, p53, and PCNA expression with treatment outcome in osteosarcoma. J Surg Oncol. (2000) 73:125-6. doi: 10.1002/(SICI)10969098(200002)73:2<125::AID-JSO14>3.0.CO;2-5

31. Wang Z, He R, Xia H, Wei YU, Wu S. MicroRNA-101 has a suppressive role in osteosarcoma cells through the targeting of c-FOS. Exp Ther Med. (2016) 11:1293-9. doi: 10.3892/etm.2016.3085

32. Gamberi G, Benassi MS, Bohling T, Ragazzini P, Molendini L, Sollazzo MR, et al. C-myc and c-fos in human osteosarcoma: prognostic value of mRNA and protein expression. Oncology (1998) 55:556-63. doi: 10.1159/000011912
33. Louis DN, Ohgaki H, Wiestler OD, Cavenee WK, Burger PC, Jouvet A, et al. The 2007 WHO classification of tumours of the central nervous system. Acta Neuropathol. (2007) 114:97-109. doi: 10.1007/s00401-007-0243-4

34. Hanahan D, Weinberg RA. Hallmarks of cancer: the next generation. Cell 2011:144:646-74. doi: 10.1016/j.cell.2011.02.013

35. Ottaviani G, Jaffe N. The epidemiology of osteosarcoma. Cancer Treat Res. (2009) 152:3-13. doi: 10.1007/978-1-4419-0284-9_1

36. Chen D, Zhao Z, Huang Z, Chen DC, Zhu XX, Wang YZ, et al. Super enhancer inhibitors suppress MYC driven transcriptional amplification and tumor progression in osteosarcoma. Bone Res. (2018) 6:11. doi: 10.1038/s41413-018-0009-8

37. Gao Q, Gu Y, Jiang Y, Fan L, Wei Z, Jin H, et al. Long non-coding RNA Gm2199 rescues liver injury and promotes hepatocyte proliferation through the upregulation of ERK1/2. Cell Death Dis. (2018) 9:602. doi: 10.1038/s41419-018-0595-9

38. Wang Y, Wan D, Zhou R, Zhong W, Lu S, Chai Y. Geraniin inhibits migration and invasion of human osteosarcoma cancer cells through regulation of PI3K/Akt and ERK1/2 signaling pathways. Anticancer Drugs (2017) 28:95966. doi: 10.1097/CAD.0000000000000535

39. Lin H, Hao Y, Zhao Z, Tong Y. Sirtuin 6 contributes to migration and invasion of osteosarcoma cells via the ERK1/2/MMP9 pathway. FEBS Open Bio (2017) 7:1291-301. doi: 10.1002/2211-5463.12265

40. Huang K, Chen Y, Zhang R, Wu Y, Ma Y, Fang X, et al. Honokiol induces apoptosis and autophagy via the ROS/ERK1/2 signaling pathway in human osteosarcoma cells in vitro and in vivo. Cell Death Dis. (2018) 9:157. doi: 10.1038/s41419-017-0166-5

41. Sears R, Leone G, DeGregori J, Nevins JR. Ras enhances Myc protein stability. Mol Cell (1999) 3:169-79. doi: 10.1016/S1097-2765(00)80308-1

42. Sears R, Nuckolls F, Haura E, Taya Y, Tamai K, Nevins JR. Multiple Rasdependent phosphorylation pathways regulate Myc protein stability. Genes Dev. (2000) 14:2501-14. doi: 10.1101/gad.836800

Conflict of Interest Statement: The authors declare that the research was conducted in the absence of any commercial or financial relationships that could be construed as a potential conflict of interest.

Copyright (c) 2019 Yu, Tang, Lin, Yao and Shen. This is an open-access article distributed under the terms of the Creative Commons Attribution License (CC BY). The use, distribution or reproduction in other forums is permitted, provided the original author(s) and the copyright owner(s) are credited and that the original publication in this journal is cited, in accordance with accepted academic practice. No use, distribution or reproduction is permitted which does not comply with these terms. 